\title{
Resonance assignment of coiled-coil 3 (CC3) domain of human STIM1
}

\author{
Agrim Gupta ${ }^{1}$ (D) Christian Manuel Kitzler ${ }^{1} \cdot$ Petr Rathner $^{2,5}$ (D) Marc Fahrner $^{3}$ (D) Herwig Grabmayr ${ }^{3}$. \\ Adriana Rathner ${ }^{2}$ (i) $\cdot$ Christoph Romanin ${ }^{3}$ (i) $\cdot$ Norbert Müller ${ }^{1,4}($ )
}

Received: 4 March 2021 / Accepted: 11 August 2021 / Published online: 21 August 2021

(c) The Author(s) 2021

\begin{abstract}
The protein stromal interaction molecule 1 (STIM1) plays a pivotal role in mediating store-operated calcium entry (SOCE) into cells, which is essential for adaptive immunity. It acts as a calcium sensor in the endoplasmic reticulum (ER) and extends into the cytosol, where it changes from an inactive (tight) to an active (extended) oligomeric form upon calcium store depletion. NMR studies of this protein are challenging due to its membrane-spanning and aggregation properties. Therefore follow the divide-and-conquer approach, focusing on individual domains first is in order. The cytosolic part is predicted to have a large content of coiled-coil (CC) structure. We report the ${ }^{1} \mathrm{H},{ }^{13} \mathrm{C},{ }^{15} \mathrm{~N}$ chemical shift assignments of the CC 3 domain. This domain is crucial for the stabilisation of the tight quiescent form of STIM1 as well as for activating the ORAI calcium channel by direct contact, in the extended active form.
\end{abstract}

Keywords Calcium channel $\cdot$ Coiled-coil structure $\cdot$ Store-operated calcium entry $\cdot$ CRAC

\section{Biological context}

Calcium ions $\left(\mathrm{Ca}^{2+}\right)$ play an essential role as second messengers in every cell. $\mathrm{Ca}^{2+}$ controls a variety of cellular processes including gene expression, cell division, neuronal signaling, muscle contraction, fertilization and apoptosis. Besides voltage-dependent and ligand-activated calcium channels, store-operated calcium entry (SOCE) is of major importance for cellular calcium entry (Berridge et al. 1995; Berridge et al. 2003; Putney 1986; Putney 2005). SOCE is mediated by specific ion channels that respond with high

Norbert Müller

norbert.mueller@jku.at

1 Institute of Organic Chemistry, Johannes Kepler University Linz, Altenbergerstrasse 69, 4040 Linz, Austria

2 Institute of Inorganic Chemistry, Johannes Kepler University Linz, Altenbergerstrasse 69, 4040 Linz, Austria

3 Institute of Biophysics, Johannes Kepler University Linz, Gruberstrasse 40, 4020 Linz, Austria

4 Faculty of Science, University of South Bohemia, Branišovská 1645/31A, 37005 České Budějovice, Czech Republic

5 Present Address: Institute of Analytical Chemistry, University of Vienna, Währingerstrasse 38, 1090 Vienna, Austria sensitivity to the depletion of the intracellular calcium stores within the endoplasmic reticulum (ER). Two key proteins form the calcium release activated calcium channel (CRAC). Orai1, which forms the $\mathrm{Ca}^{2+}$ channel in the plasma membrane, and STIM, which senses changes in ER-luminal $\mathrm{Ca}^{2+}$ concentration and controls the channel activity accordingly. The STIM protein family consists mainly of STIM1 and STIM2, with different isoforms expressed in a cell typespecific manner. STIM1 occupies the dominant role in most cell types (Roos et al. 2005; Feske et al. 2006; Prakriya et al. 2015; Fahrner et al. 2017; Grabmayr et al. 2020). In humans both STIM1 and Orai1 are associated with pathological gain-of-function $(\mathrm{GoF})$ and loss-of-function ( $\mathrm{LoF})$ mutations. These lead to severe diseases such as severe combined immunodeficiency (SCID), tubular aggregate myopathy (TAM) and the Stormorken syndrome. The symptoms of the resulting different pathological conditions represent a clinical continuum in both GoF and LoF. Depending on the mutant form, the disease courses can be mild or even lethal (Lacruz et al. 2015; Nesin et al. 2014; Morin et al. 2014; Misceo et al. 2014; Fahrner et al. 2018; Morin et al. 2020).

In recent years, an advanced model of the store-depletion triggered STIM1 activation has been developed, giving special importance to the role of both the N-terminal ER luminal and the C-terminal cytosolic part of STIM1 (Prakriya et al. 2015; Grabmayr et al. 2020; Fahrner et al. 2020). The STIM1 
$\mathrm{N}$-terminus contains the calcium sensitive EF-SAM (sterile $\alpha$ motif) domain, which senses the luminal calcium concentration. A decrease in ER calcium concentration results in conformational change of the luminal part of STIM1, signal propagation along the transmembrane domain and triggering of a massive structural change in the C-terminal STIM1 part (Fahrner et al. 2020).

The cytosolic portion of STIM1 contains three coiled coil (CC) domains denoted as $\mathrm{CC} 1, \mathrm{CC} 2, \mathrm{CC} 3$ with $\mathrm{CC} 1$ subdivided into three alpha helices $(\alpha 1, \alpha 2$ and $\alpha 3)$ (Rathner et al. 2021). The CC designation is based on bio-informatic analysis of the sequences, identifying residue repeats with high CC propensities. The basic secondary structure is $\alpha$ helical. But apparently, depending on the functional stage of the proteins different inter-helical CC-contacts can be formed. The designation CC-1, 2, 3 refers to segments of the structure which are $\alpha$ helical and separated by loops. Previous investigations imply that at least one of the functional stages is involved in an actual CC structure. The interhelical contacts may be intra- or inter-molecular in the full-length protein.

The CC2 and CC 3 domains contain the main STIM/Orai1 activating region (SOAR) also known as CRAC activating domain (CAD) which enables STIM1 to couple to and activate Orai1 (Park et al. 2009; Yuan et al. 2009).

The activation mechanism of STIM1 has been intensively investigated for more than 10 years. A tight, quiescent state and an extended activated state of STIM1 have been identified. The tight state is realized by a "clamp" between $\mathrm{CC} 1 \alpha 1$ and CC3 keeping CAD/SOAR close to the ER membrane. Upon store depletion, $\mathrm{CC} 1$ homomerization occurs, which elicits the release of the clamp, realizing the extended state. After a further oligomerization process potentially involving $\mathrm{CC} 3$, STIM1 couples to Orai1 and activates the channel (Muik et al. 2009; Fahrner et al. 2014, 2020; Rathner et al. 2021; Zhou et al. 2013).

Thus, STIM1 CC 3 is an essential domain with multiple functions: In resting cells, it stabilizes the protein in the quiescent tight state. Upon activation, it engages in STIM homo-oligomerization, supporting STIM1 cluster formation. In addition, the linker region between $\mathrm{CC} 3$ and $\mathrm{CC} 2$ is an important contact domain to Orai1, essential for channel activation (Frischauf et al. 2009; Muik et al. 2009, 2011; Fahrner et al. 2014; Zhou et al. 2013). In the present study, we report the first solution NMR resonance assignment and prediction of secondary structure propensities of the monomeric CC3 domain of wild-type human STIM1.

\section{Methods and experiments}

\section{Protein expression and purification}

The genetic information of the human STIM1 coiledcoil3 (CC3) fragment (amino acids 388-430) was amplified by PCR and cloned into a pGEX 4T-1 vector using the Xho1 and BamH1 restriction sites. The pGEX 4T-1 vector includes a GST tag along with a thrombin cleavage site. The vector was transformed into competent BL21 E. coli cells using the standard heat shock method. Transformed cells were cultivated in LB medium (ampicillin, $100 \mathrm{mg} / \mathrm{L}$ ) overnight at $37^{\circ} \mathrm{C}$. The bacterial stock was used to inoculate $250 \mathrm{~mL}$ of LB medium (1:100 dilution), the culture was further cultivated at $37^{\circ} \mathrm{C}$ until optical density (OD at 580 $\mathrm{nm})$ reached 0.8 . The cells were gently centrifuged $(2000 \mathrm{~g}$, $25^{\circ} \mathrm{C}, 20 \mathrm{~min}$ ) and resuspended in $\mathrm{M} 9$ minimal medium with $\left({ }^{15} \mathrm{NH}_{4}\right)_{2} \mathrm{SO}_{4}(1.5 \mathrm{~g} / \mathrm{L})$ and ${ }^{13} \mathrm{C}_{6}$-glucose $(3 \mathrm{~g} / \mathrm{L})$ as sole nitrogen and carbon sources, respectively and enriched with BME vitamins (Sigma Aldrich). The culture was incubated at $37{ }^{\circ} \mathrm{C}$ for another $30 \mathrm{~min}$ and then isopropyl $\beta$-D-1thiogalactopyranoside (IPTG) (final conc. $1 \mathrm{mM}$ ) was added and the temperature lowered to $28{ }^{\circ} \mathrm{C}$ before overnight cultivation. After centrifugation, the cell pellet was suspended in $20 \mathrm{mM}$ Tris-HCl, $100 \mathrm{mM} \mathrm{NaCl}, 5 \mathrm{mM} \beta$-mercaptoethanol ( $\beta \mathrm{ME}), 10 \%$ Glycerol, $0.1 \%$ Triton $\mathrm{X}-100, \mathrm{pH} 7.5$ adding 4-(2-Aminoethyl)benzenesulfonyl fluoride hydrochloride (AEBSF) $(36 \mu \mathrm{g} / \mathrm{mL}$, neoLab) as protease inhibitor. After sonication on ice ( 6 cycles of $1 \mathrm{~min}$ ), an existing purification protocol (Rathner et al. 2018) was slightly modified in order to increase the yield of soluble $\mathrm{CC} 3$ protein fragment. The homogeneity of the fragment was monitored by SDS-PAGE and the protein sample was concentrated to $0.5 \mathrm{mM}$ using Amicon ${ }^{\circledR}$ ultra centrifugal filters (Merck) with $3 \mathrm{kDa}$ cut-off and stored at $4{ }^{\circ} \mathrm{C}$.

\section{NMR spectroscopy}

The purified protein samples were swapped to $20 \mathrm{mM}$ Bis-Tris, $10 \% \mathrm{v} / \mathrm{v} \mathrm{D}_{2} \mathrm{O}, \mathrm{pH} 6.0$ and $550 \mu \mathrm{L}$ (protein concentration $0.5 \mathrm{mM}$ ) transferred to $5 \mathrm{~mm}$ NMR tubes (Wilmad 535PPT). Following previous studies, 2,2,2-trifluoroethanol (TFE) buffer additive at various concentrations was tested to suppress aggregation and obtain sufficient resolution in ${ }^{1} \mathrm{H}-{ }^{15} \mathrm{~N}$ HSQC spectra (Rathner et al. 2018; Stathopulos et al. 2013). The final resonance assignment was carried out with the addition of $17.5 \% \mathrm{v} / \mathrm{v}$ TFE at $310 \mathrm{~K}$ by the combination of common $2 \mathrm{D}$ and $3 \mathrm{D}$ experiments: ${ }^{1} \mathrm{H}-{ }^{15} \mathrm{~N}$ HSQC, ${ }^{1} \mathrm{H}-{ }^{13} \mathrm{C}$ HSQC, HNCACB, HN(CA)CO, HNCO, HNCA, $\mathrm{H}(\mathrm{CCO}) \mathrm{NH}$ and $\mathrm{CC}(\mathrm{CO}) \mathrm{NH}$ (Montelione et al. 1992; Grzesiek and Bax 1993; Sattler et al. 1999). All the NMR 
experiments were performed on a $700 \mathrm{MHz}$ Bruker Avance III spectrometer equipped with a TCI cryogenically cooled $(20 \mathrm{~K})$ probe and processed by Topspin ${ }^{\circledR}$ software (version 3.6). Backbone dihedral angles and secondary structure propensities were predicted using TALOS-N (Shen and Bax 2013). Additionally, a structure prediction with CS-Rosetta software was performed (Shen et al. 2008).

\section{Spectroscopy}

The CD spectra were obtained using the Jasco J-810 spectropolarimeter by averaging of three measurement runs (180-280 nm, $10 \mathrm{~nm} / \mathrm{min}$ scanning speed) at $20^{\circ} \mathrm{C}$ using a quartz cuvette with $0.1 \mathrm{~cm}$ path length at sample concentration of $50 \mu \mathrm{M}$ and processed with Jasco spectra manager (v. 2.14). In order to evaluate the impact of TFE on the CC3 secondary structure, the spectra were measured in 20 $\mathrm{mM}$ Bis-Tris, $\mathrm{pH} 6.0$ with and without addition of $17.5 \%$ $\mathrm{v} / \mathrm{v}$ TFE. The coiled-coil index was determined from the global and local minima at $208 \mathrm{~nm}$ and $220 \mathrm{~nm}$. The ratio $\mathrm{r}$ $=[\mathrm{CD}]_{222} /[\mathrm{CD}]_{208} \geq 1$ indicates a coiled coil motif whereas $\mathrm{r} \leq 0.86$ indicates an isolated $\alpha$ helix (Greenfield 2006).

\section{Dynamic light scattering}

Aggregation properties of isolated STIM1 CC3 were probed by dynamic light scattering (DLS) using a Zetasizer Nano ZSP 2 (Malvern). Measurements were carried out in 15 $\mathrm{mm}$ centre height UV-micro cuvettes and were performed by averaging 6 runs (with automatic optimized repetitions of $10 \mathrm{~s}$ measurements) for both $\mathrm{CC} 3$ in $20 \mathrm{mM}$ Bis-Tris and CC3 in $20 \mathrm{mM}$ Bis-Tris, $17.5 \% \mathrm{v} / \mathrm{v}$ TFE at $20^{\circ} \mathrm{C}$. The analysis was performed using the Zetasizer Software version 8.01 (Malvern).

\section{Extent of assignments and data deposition}

${ }^{1} \mathrm{H}-{ }^{15} \mathrm{~N}$ Heteronuclear single quantum coherence spectroscopy (HSQC) and transverse relaxation-optimized spectroscopy (TROSY-HSQC) experiments were first run at $298 \mathrm{~K}$ in $20 \mathrm{mM}$ Bis-Tris at pH 6.0. Extensive signal overlap presumably due to coiled-coil aggregation of the domain (see coiled-coil formation prediction in Supplementary Data Fig. S1), and sample instability made it impossible to find a sufficient number of assignable resonances. In order to reduce peak overlap and increase resolution in the HSQC-type spectra, measurements at lower protein concentration as well as $\mathrm{pH}$ screening including various buffer components $(\mathrm{NaCl}$, HEPES, etc.) were carried out. However, there was no significant improvement in the spectral resolution as documented in the Supplementary Data Fig. S2 a). Only with the increasing concentration of TFE the number of observable resonances increased (Fig. S2 b-e). Finally, the aggregation tendency of the CC3 domain was overcome upon addition of $17.5 \% \mathrm{v} / \mathrm{v} 2,2,2$-TFE at $310 \mathrm{~K}$. These conditions had successfully been used in a previous NMR study of STIM1 coiled coil fragments (Stathopulos et al. 2013), where TFE was shown to be an excellent additive to dissociate strongly aggregating coiled coil polypeptides. While preserving the tertiary structure intact, it enables protein-protein interaction studies in solution without high-order oligomerization. From previous experimental NMR in vitro and in vivo studies it appears that in presence of ionic detergents or organic solvents (TFE) stable monodisperse solutions of the STIM cytosolic fragments are observed which exhibit close structural similarities to the active form of functional full length STIM1 (Stathopulos et al. 2013; Rathner et al. 2021). In the absence of TFE the isolated CC3 structure apparently "saturates" the missing intramolecular coiled-oil contacts within full-length STIM1 by homo-oligomerization. Thus the use of an additive for the $\mathrm{CC} 3$ fragment serves the purpose of avoiding $\mathrm{CC} 3-\mathrm{CC} 3$ coiled-coil interactions, because in full-length STIM1 the CC contacts occur between different domains.

Under the final conditions the $2 \mathrm{D}{ }^{1} \mathrm{H}-{ }^{15} \mathrm{~N}$ HSQC spectrum shows well resolved resonances for all 43 residues (Fig. 1). We have assigned $99.5 \%$ of the backbone resonances which include all of ${ }^{1} \mathrm{HN},{ }^{15} \mathrm{~N},{ }^{13} \mathrm{C} \alpha,{ }^{13} \mathrm{CO}$ and $97.7 \%$ of the $\mathrm{H} \alpha$ resonances. Side-chain assignment includes fully assigned resonances of ${ }^{13} \mathrm{C} \beta$ followed by $95.4 \%$ of $\mathrm{H} \beta$, $90.3 \%$ of $\mathrm{C} \gamma, 80.9 \%$ of $\mathrm{H} \gamma, 35.48 \%$ of $\mathrm{C} \delta, 40 \%$ of $\mathrm{H} \delta$ and $24 \%$ of $\mathrm{H} \varepsilon$. The aromatic residues have not been assigned yet. These assignments serve as a basis for future interaction studies between different domains within STIM1. The assigned chemical shifts have been deposited into BioMagResBank (http://www.bmrb.wisc.edu) with accession number 50683 .

\section{Structure prediction}

From the chemical shift data of ${ }^{13} \mathrm{C} \alpha,{ }^{13} \mathrm{C} \beta,{ }^{13} \mathrm{CO},{ }^{1} \mathrm{H} \alpha,{ }^{1} \mathrm{HN}$ and ${ }^{15} \mathrm{~N}$ shifts the secondary structure propensities using TALOS-N web-based software were obtained (Fig. 2a; Shen and Bax 2013). The chemical shift data were used for the generation of 10,000 structures using CS-Rosetta (Shen et al. 2008). The structure with the smallest ratio of lowest energy and RMSD was selected and is displayed in Fig. 2b. The main structural features are a short (single turn) $\alpha$-helix at the $\mathrm{N}$-terminus followed by approximately 10 amino acids in a disordered loop region (H395 to D404) and a longer (ca. 6 turn) $\alpha$-helix (V405 to L427). 
Fig. 1 Assigned ${ }^{1} \mathrm{H}_{-}{ }^{15} \mathrm{~N}$ HSQC spectrum of human wild-type STIM1 CC3 acquired in $20 \mathrm{mM}$ Bis-Tris, $17.5 \%$ TFE, pH 6.0 at $310 \mathrm{~K}$ at a protein concentration of $0.5 \mathrm{mM}$. The peaks labelled with asterisks $(*)$ originate from side-chain $\mathrm{N}-\mathrm{H}$ correlations of Trp430, Asn388 and Gln414

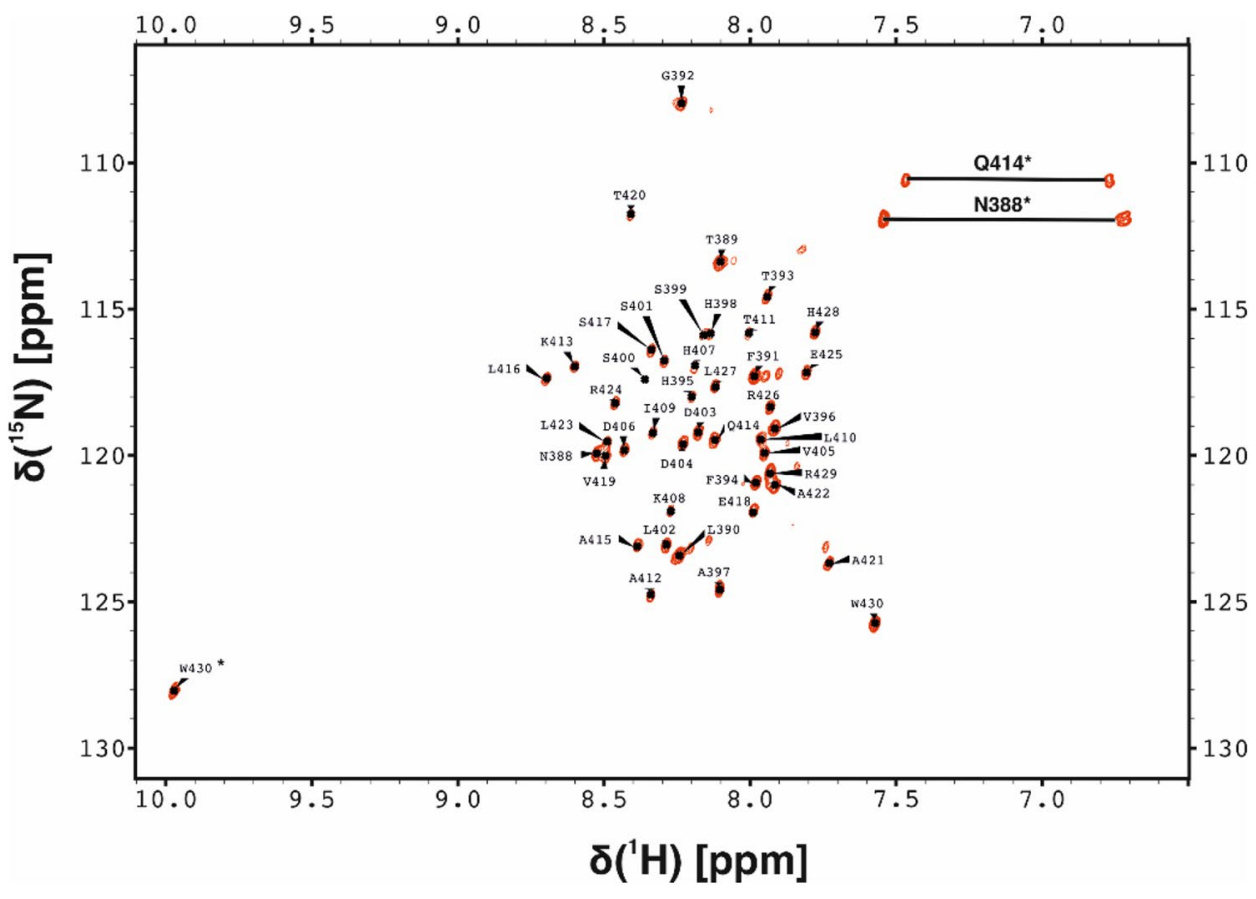

Fig. 2 Secondary structure prediction for STIM1 CC3 a Results from TALOS-N (Shen and Bax 2013). Blue lines represent the order parameter RCI-S ${ }^{2}$ predicted from the chemical shifts. Black bars indicate secondary structure propensities for each residue. b Three-dimensional structural model of the monomeric STIM1 CC3 fragment from CS-Rosetta
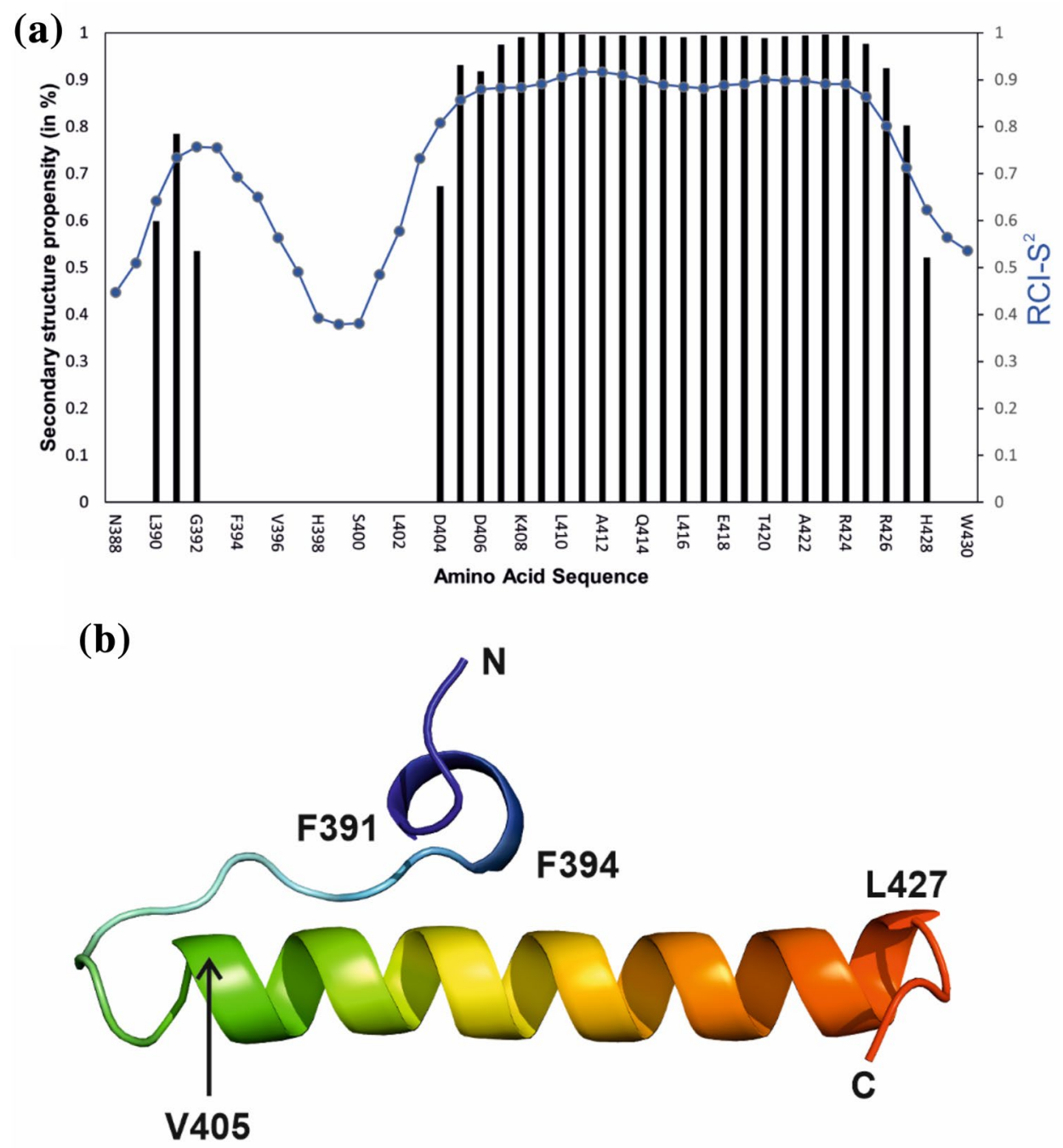
Fig. 3 CD spectra of $0.5 \mathrm{mM}$ STIM1 CC3 WT in absence (red) and presence of (blue) $17.5 \% \mathrm{v} / \mathrm{v}$ TFE at $20{ }^{\circ} \mathrm{C}$

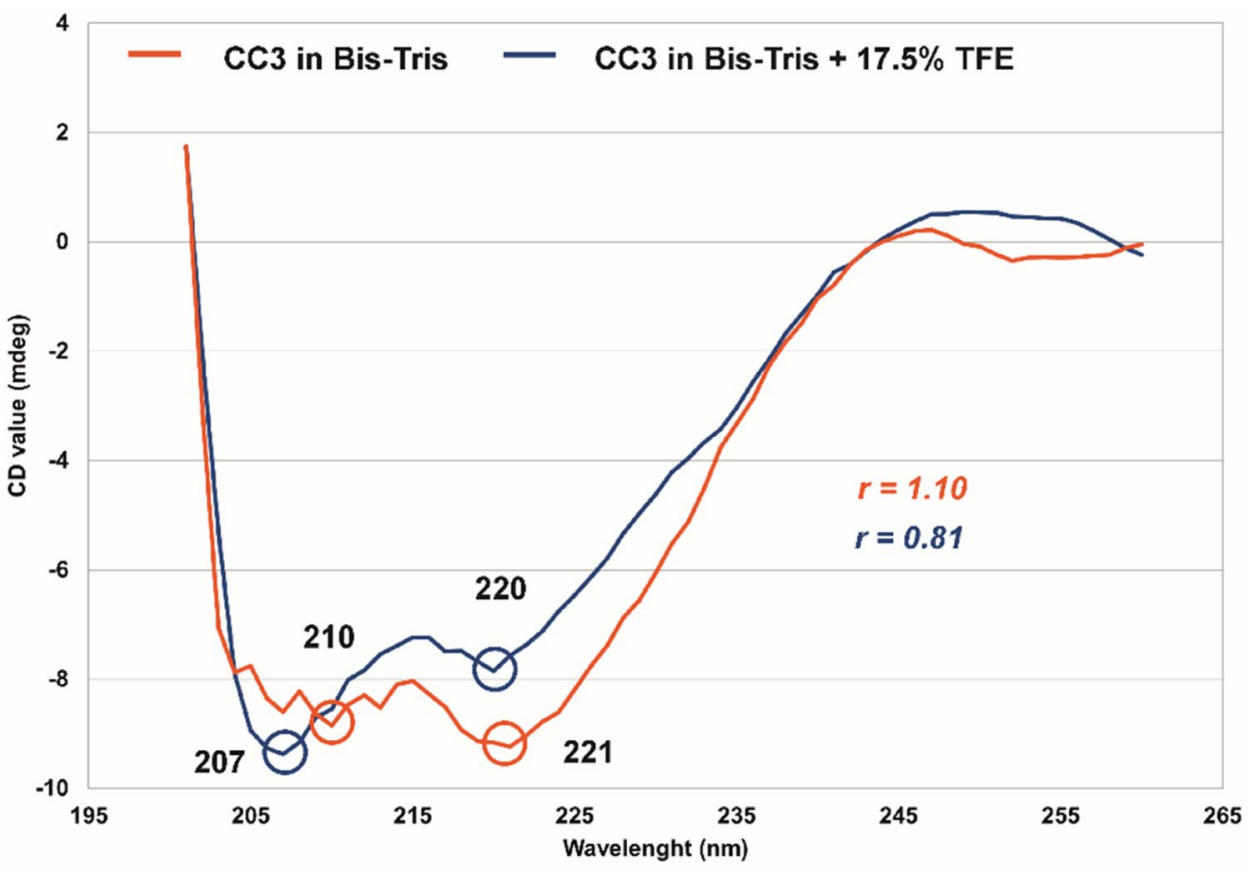

\section{CD spectroscopy}

The CD profile (Fig. 3) of STIM1 CC3 corresponds to a protein with high $\alpha$ helical content. The ratio $r$ of $[\mathrm{CD}]_{222 \mathrm{~nm}} /$ $[\mathrm{CD}]_{208 \mathrm{~nm}}$ without the TFE is 1.10 , which is clear evidence that STIM1 CC3 forms predominantly coiled-coil oligomers. By contrast, in the presence of 2,2,2-TFE, the $\alpha$ helical $\mathrm{CD}$ profile remained but the $[\mathrm{CD}]_{222 \mathrm{~nm}} /[\mathrm{CD}]_{208 \mathrm{~nm}}$ ratio was reduced to 0.81 which is characteristic of isolated $\alpha$ helix (Greenfield 2006).

\section{Dynamic light scattering}

DLS scans of free CC3 in $20 \mathrm{mM}$ Bis-Tris in the absence of TFE showed existence of higher order oligomers (Fig. S3 a). The population was classified as polydisperse with the major mass maximum around $443 \mathrm{kDa}$. The calculated radius was $7.84 \mathrm{~nm}$ at $20^{\circ} \mathrm{C}$ for the aggregated sample in the absence of TFE. Repetition of the same experiment with addition of $17.5 \% \mathrm{v} / \mathrm{v}$ TFE resulted in much smoother correlation decay curve. However, the exact determination of the protein size and weight was hindered by the presence of the TFE cosolvent itself (Fig. S3 b) (Gast and Modler 2007).

\section{Conclusions}

We have reported complete backbone and near-complete side chain NMR resonance assignments of the human wildtype STIM1 CC3 domain. Based on these data, we obtained a prediction of its secondary structure propensities along with its tertiary structure model in the presence of 2,2,2-trifluoroethanol. As expected from the coiled-coil characteristics of the primary sequence, the monomeric isolated STIM1 CC3 domain is predominantly $\alpha$-helical, if the coiled-coil interactions are suppressed by a suitable co-solvent. In the monomeric state, a short disordered segment (H395 to D404) interrupts the $\alpha$-helix close to the $\mathrm{N}$-terminus. These data will serve as a basis for further structural and in vivo functional studies of the STIM1 activation mechanism, for which coiled-coil interaction moieties in the $\mathrm{CC} 3$ domain are crucial (Fahrner et al. 2018).

Supplementary Information The online version contains supplementary material available at https://doi.org/10.1007/s12104-021-10042-7.

Acknowledgements We gratefully acknowledge the help of Dr. Yolanda Salinas Soler (Institute of Polymer Chemistry, JKU Linz) with the DLS measurements. This work has been supported by the Austrian Science Fund (FWF) PhD program W1250 'NanoCell', P32947 to M.F. and P27263 to C.R. The NMR experiments were performed at the NMR laboratory of the Upper Austrian-South-Bohemian Research Infrastructure Center in Linz, 'RERI-uasb', supported by the European Union through the ERDF INTERREG IV (RU2-EU-124/100-2010) program (ETC Austria-Czech Republic 2007-2013, Project M00146, 'RERI-uasb', for N.M.)

Author contributions NM and CR conceived the project. PR and AR planned and supervised the expression and NMR experiments. MF and $\mathrm{HG}$ produced the transformed plasmids. AG and CMK expressed, isolated and purified the protein samples for NMR experiments. AG acquired and analyzed the data under the supervision of PR and AR. All the authors contributed to writing the manuscript and figures.

Funding This work has been supported by the Austrian Science Fund (FWF) PhD program W1250 'NanoCell', as well as project 
numbers P32947 and P27263. The NMR laboratory of the Upper Austrian-South-Bohemian Research Infrastructure Center in Linz was supported by the European Union through the ERDF INTERREG IV (RU2-EU-124/100-2010) program (ETC Austria-Czech Republic 2007-2013, Project M00146, 'RERI-uasb'). Open access funding provided by Johannes Kepler University Linz.

Data availability The chemical shift values for the ${ }^{1} \mathrm{H},{ }^{13} \mathrm{C}$ and ${ }^{15} \mathrm{~N}$ resonances have been deposited in the BioMagResBank (https://www. bmrb.wisc.edu) under Accession Number 50683.

\section{Declarations}

Conflict of interest The authors declare no conflict of interest.

Open Access This article is licensed under a Creative Commons Attribution 4.0 International License, which permits use, sharing, adaptation, distribution and reproduction in any medium or format, as long as you give appropriate credit to the original author(s) and the source, provide a link to the Creative Commons licence, and indicate if changes were made. The images or other third party material in this article are included in the article's Creative Commons licence, unless indicated otherwise in a credit line to the material. If material is not included in the article's Creative Commons licence and your intended use is not permitted by statutory regulation or exceeds the permitted use, you will need to obtain permission directly from the copyright holder. To view a copy of this licence, visit http://creativecommons.org/licenses/by/4.0/.

\section{References}

Berridge MJ (1995) Capacitative calcium entry. Biochem J 312(Pt 1):1-11. https://doi.org/10.1042/bj3120001

Berridge MJ, Bootman MD, Roderick HL (2003) Calcium signalling: dynamics, homeostasis and remodelling. Nat Rev Mol Cell Biol 4:517-529. https://doi.org/10.1038/nrm1155

Fahrner M, Muik M, Schindl R et al (2014) A coiled-coil clamp controls both conformation and clustering of stromal interaction molecule 1 (STIM1). J Biol Chem 289:33231-33244. https://doi.org/ 10.1074/jbc.M114.610022

Fahrner M, Schindl R, Muik M et al (2017) The STIM-Orai pathway: the interactions between STIM and Orai. Adv Exp Med Biol 993:59-81. https://doi.org/10.1007/978-3-319-57732-6_4

Fahrner M, Stadlbauer M, Muik M et al (2018) A dual mechanism promotes switching of the Stormorken STIM1 R304W mutant into the activated state. Nat Commun 9:825. https://doi.org/10. 1038/s41467-018-03062-w

Fahrner M, Grabmayr H, Romanin C (2020) Mechanism of STIM activation. Curr Opin Physiol 17:74-79. https://doi.org/10.1016/j. cophys.2020.07.006

Feske S, Gwack Y, Prakriya M et al (2006) A mutation in Orai1 causes immune deficiency by abrogating CRAC channel function. Nature 441:179-185. https://doi.org/10.1038/nature04702

Frischauf I, Muik M, Derler I et al (2009) Molecular determinants of the coupling between STIM1 and Orai channels: differential activation of Orai1-3 channels by a STIM1 coiled-coil mutant. J Biol Chem 284(32):21696-21706. https://doi.org/10.1074/jbc. M109.018408

Gast K, Modler AJ (2007) Dynamic and static light scattering of Proteins. In: Uversky VN, Permyakov EA (eds) Methods in protein structure and stability analysis. Nova Science Publishers, Inc, NewYork, pp 281-316
Grabmayr H, Romanin C, Fahrner M (2020) STIM proteins: an everexpanding family. Int J Mol Sci 22(1):E378

Greenfield NJ (2006) Using circular dichroism spectra to estimate protein secondary structure. Nat Protoc 1:2876-2890. https://doi.org/ 10.1038/nprot.2006.202

Grzesiek S, Bax A (1993) Methodological advances in protein NMR. Acc Chem Res 26(4):131-138. https://doi.org/10.1021/ar000 $28 \mathrm{a} 001$

Lacruz RS, Feske S (2015) Diseases caused by mutations in ORAI1 and STIM1. Ann N Y Acad Sci 1356:45-79. https://doi.org/10. 1111/nyas.12938

Misceo D, Holmgren A, Louch WE et al (2014) A dominant STIM1 mutation causes Stormorken syndrome. Hum Mutat 35:556-564. https://doi.org/10.1002/humu.22544

Morin G, Bruechle NO, Singh AR et al (2014) Gain-of-function mutation in STIM1 (p.R304W) is associated with Stormorken syndrome. Hum Mutat 35:1221-1232. https://doi.org/10.1002/humu. 22621

Morin G, Biancalana V, Echaniz-Laguna A et al (2020) Tubular aggregate myopathy and Stormorken syndrome: mutation spectrum and genotype/phenotype correlation. Hum Mutat 41:17-37. https:// doi.org/10.1002/humu.23899

Muik M, Fahrner M, Derler I et al (2009) A cytosolic homomerization and a modulatory domain within STIM1 C terminus determine coupling to ORAI1 channels. J Biol Chem 284:8421-8426. https://doi.org/10.1074/jbc.C800229200

Muik M, Fahrner M, Scindl R et al (2011) STIM1 couples to ORAI1 via an intramolecular transition into an extended conformation. EMBO J 30:1678-1689. https://doi.org/10.1038/emboj.2011.79

Nesin V, Wiley G, Kousi M et al (2014) Activating mutations in STIM1 and ORAI1 cause overlapping syndromes of tubular myopathy and congenital miosis. Proc Natl Acad Sci USA 111:4197-4202. https://doi.org/10.1073/pnas.1312520111

Park CY, Hoover PJ, Mullins FM et al (2009) STIM1 clusters and activates CRAC channels via direct binding of a cytosolic domain to Orai1. Cell 136:876-890. https://doi.org/10.1016/j.cell.2009. 02.014

Prakriya M, Lewis RS (2015) Store-operated calcium channels. Physiol Rev 95:1383-1436. https://doi.org/10.1152/physrev.00020.2014

Putney JW Jr (1986) A model for receptor-regulated calcium entry. Cell Calcium 7:1-12. https://doi.org/10.1016/0143-4160(86)90026-6

Putney JW Jr (2005) Capacitative calcium entry: sensing the calcium stores. J Cell Biol 169:381-382. https://doi.org/10.1083/jcb.20050 3161

Rathner P, Stadlbauer M, Romanin C et al (2018) Rapid NMR-scale purification of ${ }^{15} \mathrm{~N},{ }^{13} \mathrm{C}$ isotope-labeled recombinant human STIM1 coiled-coil fragments. Protein Expr Purif 146:45-50. https://doi.org/10.1016/j.pep.2018.01.013

Rathner P, Fahrner M, Cerofolini L et al (2021) Interhelical interactions within the STIM1 CC1 domain modulate CRAC channel activation. Nat Chem Biol 17:196-204. https://doi.org/10.1038/ s41589-020-00672-8

Roos J, DiGregorio PJ, Yeromin AV et al (2005) STIM1, an essential and conserved component of store-operated $\mathrm{Ca} 2+$ channel function. J Cell Biol 169(3):435-445. https://doi.org/10.1083/jcb. 200502019

Sattler M, Schleucher J, Griesinger C (1999) Heteronuclear multidimensional NMR experiments for the structure determination of proteins in solution employing pulsed field gradients. Prog Nucl Magn Reson Spectrosc 34:93-158

Shen Y, Bax A (2013) Protein backbone and sidechain torsion angles predicted from NMR chemical shifts using artificial neural networks. J Biomol NMR 56:227-241. https://doi.org/10.1007/ s10858-013-9741-y

Shen Y, Lange O, Delaglio F et al. (2008) Consistent blind protein structure generation from NMR chemical shift data. Proc Natl 
Acad Sci 105(12): 4685-4690. https://doi.org/10.1073/pnas.08002 56105

Stathopulos PB, Schnidl R, Fahrner M et al (2013) STIM1/Orai1 coiled-coil interplay in the regulation of store-operated calcium entry. Nat Commun 4:2963. https://doi.org/10.1038/ncomms3963

Yuan JP, Zeng W, Dorwart MR et al (2009) SOAR and the polybasic STIM1 domains gate and regulate Orai channels. Nat Cell Biol 11:337-343. https://doi.org/10.1038/ncb1842
Zhou Y, Srinivasan P, Razavi S et al (2013) Initial activation of STIM1, the regulator of store-operated calcium entry. Nat Struct Mol Biol 20:973-981. https://doi.org/10.1038/nsmb.2625

Publisher's Note Springer Nature remains neutral with regard to jurisdictional claims in published maps and institutional affiliations. 